\title{
Colorectal cancer cell-derived microRNA200 modulates the resistance of adjacent blood endothelial barriers in vitro
}

\author{
SILVIO HOLZNER ${ }^{1}$, DANIEL SENFTER ${ }^{1}$, SERENA STADLER ${ }^{1}$, ANNA STARIBACHER ${ }^{2}$, \\ CHI HUU NGUYEN ${ }^{1,3}$, ANNA GAGGL ${ }^{1}$, SILVANA GELEFF ${ }^{1}$, NICOLE HUTTARY ${ }^{1}$, SIGURD KRIEGER ${ }^{1}$, \\ WALTER JÄGER ${ }^{3}$, HELMUT DOLZNIG $^{4}$, ROBERT M. MADER $^{2}$ and GEORG KRUPITZA ${ }^{1}$ \\ ${ }^{1}$ Clinical Institute of Pathology, Medical University of Vienna; ${ }^{2}$ Department of Medicine I, Comprehensive Cancer Centre, \\ Medical University of Vienna; ${ }^{3}$ Department of Clinical Pharmacy and Diagnostics, University of Vienna; \\ ${ }^{4}$ Institute of Medical Genetics, Medical University of Vienna, A-1090 Vienna, Austria
}

Received May 4, 2016; Accepted September 2, 2016

DOI: $10.3892 /$ or.2016.5114

\begin{abstract}
Since cancer cells, when grown as spheroids, display drug sensitivity and radiation resistance patterns such as seen in vivo we recently established a three-dimensional (3D) in vitro model recapitulating colorectal cancer (CRC)-triggered lymphatic endothelial cell (LEC)-barrier breaching to study mechanisms of intra-/extravasation. CRC metastasizes not only through lymphatics but also through blood vessels and here we extend the 3D model to the interaction of blood endothelial cells (BECs) with naïve and 5-fluorouracil (5-FU)-resistant CRC CCL227 cells. The 3D model enabled quantifying effects of tumour-derived microRNA200 (miR200) miR200a, miR200b, miR200c, miR141 and miR429 regarding the induction of so-called 'circular chemorepellent-induced defects' (CCIDs) within the BEC-barrier, which resemble gates for tumour transmigration. For this, miR200 precursors were individually transfected and furthermore, the modulation of ZEB family expression was analysed by western blotting. miR200c, miR141 and miR429, which are contained in exosomes from naïve CCL227 cells, downregulated the expression of ZEB2, SNAI and TWIST in BECs. The exosomes of 5-FU-resistant CCL227-RH cells, which are devoid of miR200, accelerated CCID formation in BEC monolayers as compared to exosomes from naïve CCL227 cells. This confirmed the reported role of ZEB2 and SNAI in CRC metastasis and highlighted the active contribution of the stroma in the metastatic process. CCL227 spheroids affected the integrity of BEC and LEC barriers alike, which was in agreement with the observation that CRC metastasizes via blood stream (into the liver)
\end{abstract}

Correspondence to: Professor Georg Krupitza, Clinical Institute of Pathology, Medical University of Vienna, Währinger Gürtel 18-20, A-1090 Vienna, Austria

E-mail: georg.krupitza@meduniwien.ac.at

Key words: colorectal cancer, microRNA200, exosomes, intercellular epigenetics, blood endothelial barrier breaching, drug resistance as well as via lymphatics (into lymph nodes and lungs). This further validated the $\mathrm{CRC} / \mathrm{LEC}$ and $\mathrm{CRC} / \mathrm{BEC}$ in vitro model to study mechanisms of CRC spreading through vascular systems. Treatment of CCL227-RH cells with the HDAC inhibitors mocetinostat and sulforaphane reduced CCID formation to the level triggered by naïve CCL227 spheroids, however, without significantly influencing miR200 expression in CCL227-RH cells.

\section{Introduction}

The formation of metastases comprises multiple sequential steps by which malignant cells disseminate from the primary tumour, invade the surrounding stroma, breach the vasculature, travel through the fluids, extravasate and colonise distant organs (1). Colorectal cancer (CRC) and breast cancer colonise distant organs through the lymphatic vasculature. Whereas CRC frequents also blood vessels for metastatic spreading, it is widely accepted that this route in not common for breast cancer. A validated in vitro assay recapitulates the preference of breast cancer cell spheroids to breach the lymphatic endothelial cell (LEC) barrier but not the blood endothelial cell (BEC) barrier (2). The quantitative assay was further developed to study the malignant potential of CRC spheroids formed by CCL227 cells, or by the 5-fluorouracil (5-FU)-resistant (5-FU is a standard drug for CRC treatment) subclone CCL227-RH (3), to study breaching of the BEC barrier and to define potential intervention strategies. Acquisition of drug resistance is a complex process that profoundly alters gene expression at the genetic and epigenetic level and impacts on cell behaviour such as epithelial-to-mesenchymal transition (EMT) and cell mobility. Reportedly, 5-FU-resistant CRC cells breach LEC barriers significantly faster than naïve CRC cells (4). This is due to low levels of microRNA200 (miR200) in those exosomes secreted by 5-FU-resistant CCL227-RH cells (5). The exosome-encapsulated miR200 family includes five members encoded by two genes on chromosome 1 (miR200b, miR200a, miR429) and chromosome 12 (miR200c, miR141) (6) which downregulate the expression of the ZEBs and are inducers of EMT being a key process associated with the progression of CRC $(7,8)$. CRC-derived miR200 is taken up by adjacent 
LECs, which suppresses ZEB transcription factors thereby reducing plasticity and migration of LEC and slowing down the formation of gates within the LEC barrier through which the tumour can traverse (4). Therefore, metastasis formation is not only an active process of the malignant cell, but also an orchestrated and transient takeover of the endothelial barrier.

Since CRC colonises distant organs through both, lymphatics and blood vessels, we attempted to further validate the new CRC model and tested how BECs respond to naïve and resistant CRC spheroids and examined which of miR200 family members impose the strongest effect on the ZEB expression in BECs.

\section{Materials and methods}

Reagents and antibodies. Sulforaphane was from SigmaAldrich Chemie GmbH (Munich, Germany; R,S-sulforaphane, a racemic mixture of $\mathrm{R}$ and $\mathrm{S}$ isomers; the $\mathrm{R}$ isomer is biologically active), mocetinostat was from Chemietek (Indianapolis, IN, USA). Rabbit anti-zinc finger E-box-binding homeobox 1 (ZEB1), and goat anti-SLUG were from Santa Cruz Biotechnology, Inc. (Heidelberg, Germany). Rabbit anti-ZEB2 was from Merck Millipore (Darmstadt, Germany), mouse anti-VE-cadherin was from Beckman Coulter (Fullerton, CA, USA), rabbit anti-TWIST was from Abcam and rabbit anti-SNAI was from Cell Signaling Technology, Inc. (Danvers, MA, USA). Monoclonal mouse anti- $\beta$-actin (clone AC-15) was from Sigma-Aldrich Chemie $\mathrm{GmbH}$ and peroxidase-conjugated polyclonal swine anti-rabbit $\operatorname{IgG}(1: 5,000)$, peroxidase-conjugated polyclonal rabbit anti-mouse IgG (1:10,000), peroxidase-conjugated polyclonal rabbit anti-goat IgG $(1: 10,000)$ were from DakoCytomation (Glostrup, Denmark).

Cell culture. The CRC cell line CCL227 (lymph node metastasis) was purchased from the American Type Culture Collection (Rockville, MD, USA). From this cell line the highly 5-FU-resistant subclone CCL227-RH (resistant against $125 \mu \mathrm{M} 5$-FU) was selected by continuous addition of 5-FU over a period of time. Only when used for experiments 5-FU was withdrawn from cell cultures several days before. All cells were grown in RPMI-1640 medium supplemented with $10 \%$ heat-inactivated fetal calf serum, $25 \mathrm{mg} / \mathrm{ml}$ gentamycin (G418) (all from Gibco, Karlsruhe, Germany) and maintained in humidified atmosphere containing $5 \% \mathrm{CO}_{2}$ at $37^{\circ} \mathrm{C}$. The subclone CCL227-RH was continuously grown in the presence of 5-FU. Telomerase-immortalised human BECs were grown in EGM-2 MV (EBM2-based medium CC-3156 and supplement CC-4147; Clonetics, Allendale, NJ, USA) and G418. BECs were generated from human dermal microvascular endothelial cells (C-12260) obtained from PromoCell $\mathrm{GmbH}$ (Heidelberg, Germany) and telomerase-immortalized as described earlier (9).

Spheroid formation. A total of $2 \times 10^{4}$ cells $/ \mathrm{ml}(2,000$ cells/spheroid) of the cell lines CCL227 or CCL227-RH was transferred to RPMI medium containing $20 \%$ methylcellulose solution (final concentration $0.3 \%$ ). One hundred and fifty microliters of this cell suspension was added to each well of a 96-well plate (CELLSTAR 650185; Greiner Bio-One,
Kremsmünster, Austria) and centrifuged $15 \mathrm{~min}$ at 1,200 rpm to allow spheroid formation within 6 days at $37^{\circ} \mathrm{C}$ and $5 \% \mathrm{CO}_{2}$. To generate more stable and compact spheroids $1.7 \%$ Matrigel was added.

Circular chemorepellent-induced defect (CCID) assay. In this assay the sizes of the cell-free areas forming directly underneath the CRC cell spheroids in the endothelial monolayer (CCIDs) were measured. BECs were seeded into 24-well plates and grown to $\sim 90 \%$ confluence. Then, they were incubated with a 1:5,000 dilution of cyto-tracker green (Molecular Probes Life Technologies, Carlsbad, CA, USA) for $1 \mathrm{~h}$ in EGM2V medium. Spheroids were washed and transferred to the cyto-tracker-stained BEC monolayer. After $3 \mathrm{~h}$ of co-cultivation, the size of CCID areas underneath CRC cell spheroids were photographed using a fluorescence microscope Axiovert and calculated with the AxioVison Rel. 4.8 program (both from Carl Zeiss AG, Jena, Germany). The CCID areas underneath 10 or more spheroids were measured.

Spheroid treatment with inhibitors. CRC cell spheroids were collected and washed twice with fresh medium to remove methylcellulose. Then the spheroids were pre-treated with different inhibitors for $30 \mathrm{~min}$ in $1 \mathrm{ml}$ EGM2V medium. All inhibitors were dissolved fresh in DMSO and diluted to the indicated final concentrations. The DMSO concentration of $0.1 \%$ was kept constant. Afterwards, $1 \mathrm{ml}$ medium and spheroids were transferred to confluent cyto-tracker-stained BEC monolayers and incubated for $3 \mathrm{~h}$.

Western blotting. BECs were seeded in T-25 flasks and grown until $80 \%$ confluence. BECs were either transfected with the miR200 family precursors (5 $\mathrm{nM}$ of miR200a, miR200b, miR200c, miR141, and miR429) for $24 \mathrm{~h}$ or treated with drugs for $0.5,1,2,4 \mathrm{~h}$. Then, cells were washed twice with cold PBS, placed on ice and lysed with the 2X SDS lysis buffer and sonication on ice. The protein concentration was determined by Bio-Rad assay and measured with a photometer at $260 \mathrm{~nm}$. Equal protein amounts were mixed with $5 \mathrm{X}$ loading dye and lysis buffer to a final volume of $20 \mu \mathrm{l}$, denatured at $95^{\circ} \mathrm{C}$ for $5 \mathrm{~min}$ and electrophoretically separated by $10 \%$ polyacrylamide SDS gels, electrotransferred to PVDF membranes (GE Healthcare Life Sciences, Little Chalfont, UK) as described earlier (10) and immunoblots were visualized using ECL detection kit (Thermo Scientific, Portsmouth, IL, USA) and Amersham Hyperfilm (GE Healthcare, Buckinghamshire, UK).

MicroRNA transfection. BECs were transfected using siPORT NeoFX (Ambion Life Technologies, Carlsbad, CA, USA) at a confluence of $\sim 80 \%$. CRC cell spheroids were cultivated for 6 days when they were transfected. All microRNA precursors (miR200a, miR200b, miR200c, miR141, miR429) and non-targeting control RNA were diluted (in $100 \mu \mathrm{l}$ ) to a final concentration of $5 \mathrm{nM}$ in RPMI or EGM2V without serum and antibiotics. A total of $10 \mu \mathrm{l}$ siPORT reagent was mixed with $90 \mu$ l of RPMI and mixed 1:1 with the diluted microRNA solution. Thereafter, the solution was incubated for $10 \mathrm{~min}$ at room temperature. The mixture was gently added to either the cells or the spheroids in $2.3 \mathrm{ml}$ RPMI medium and after $24 \mathrm{~h}$ the medium was changed to complete RPMI medium. 
A

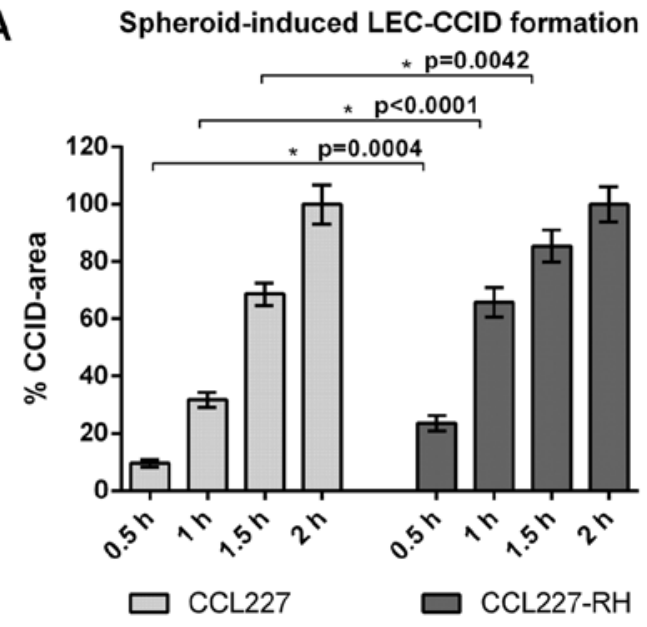

C

BEC-CCID formation upon exposure to CCL227 spheroids that were treated with exosomes from CCL227-RH cells

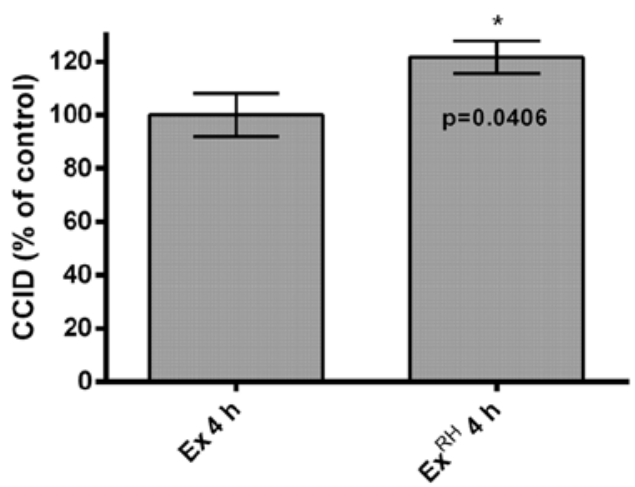

B

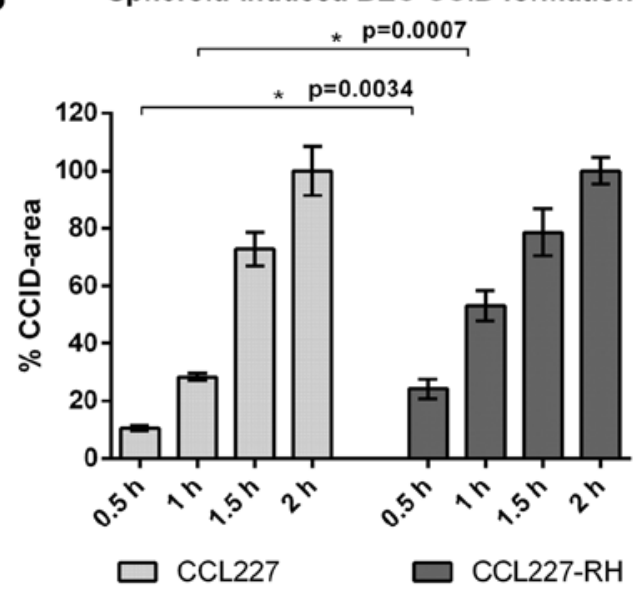

D

BEC-CCID formation upon exposure to CCL227-RH spheroids that were treated with exosomes from CCL227 cells

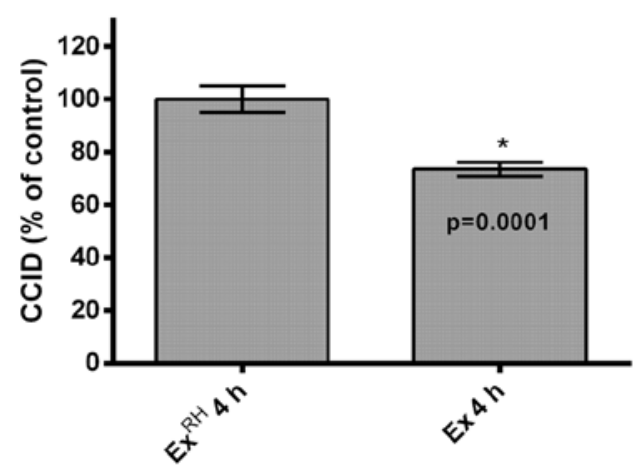

Figure 1. Distinct malignant properties of naïve CCL227 and 5-FU-high resistant CCL227-RH colon cancer phenotypes. Comparison of spheroid-induced CCID formation in (A) LEC and (B) BEC monolayers after 0.5, 1, 1.5 and $2 \mathrm{~h}$; (n=3). (C) CCL227 and (D) CCL227-RH spheroids were each pre-incubated with $100 \mu \mathrm{g}$ exosomal protein derived from naïve (Ex) and resistant (Ex $\left.{ }^{\mathrm{RH}}\right)$ CCL227 cells for $30 \mathrm{~min}$ and co-cultivated with BECs for $4 \mathrm{~h}$ and then CCID formation was measured; $(n=3)$. Error bars indicate means \pm SEM and asterisks indicate significance compared to control or to the experimental points connected by brackets ( $\mathrm{p}<0.05$; Student's t-test). 5-FU, 5-fluorouracil; CCID, circular chemorepellent-induced defect; LEC, lymphatic endothelial cell; BEC, blood endothelial cells.

Isolation of exosomes. CRC cells were grown in a T-75 flask until $\sim 80 \%$ of confluence. Afterwards, cells were washed with PBS and kept in RPMI without FCS and antibiotics for $24 \mathrm{~h}$ when the medium was changed to RPMI with $10 \%$ exosome-free FCS for further $24 \mathrm{~h}$ to allow exosome production. Thereafter, the supernatant was collected, centrifuged at $3,000 \mathrm{x} \mathrm{g}$ for $15 \mathrm{~min}$ and transferred to a new $15-\mathrm{ml}$ tube. The exosomes in the supernatant were isolated using $2 \mathrm{ml}$ ExoQuick-TC (System Biosciences, Mountain View, CA, USA) per $5 \mathrm{ml}$ supernatant and pelleted at $1,500 \mathrm{~g}, 4^{\circ} \mathrm{C}$ for $30 \mathrm{~min}$ and washed twice. Afterwards the pellet was resuspended in 10-100 $\mu 1$ DEPC water. The exosomal protein concentration was measured with a photometric protein assay (Bio-Rad, Hercules, CA, USA).

Statistical analysis. Dose-response curves were analysed using Excel 2013 software and GraphPad Prism 6 software package (GraphPad, Software, Inc., San Diego, CA, USA). The values were expressed as means \pm SEM and significance was calculated by Student's t-test and ANOVA (statistical significance $\mathrm{p}<0.05)$.

\section{Results}

Comparison of the in vitro responses of LECs and BECs to $C R C$ clones. MCF-7 and MDA-MB231 breast cancer spheroids trigger the retraction of adjacent LEC monolayers which causes the formation of CCIDs $(10,11)$. A similar centrifugal migration of LECs and BECs was seen in the CRC model and time-course experiments were performed to investigate the activity of CCL227 spheroid-triggered breaching of the beneath growing LEC and BEC barriers. 5-FU treatment of CRC causes the development of drug resistance and also the aggressiveness of tumours increases. Therefore, it was investigated whether spheroids of the highly 5-FU-resistant CCL227-RH subclone exhibited an elevated potential in breaching endothelial barriers as compared to naïve CCL227 spheroids. In fact, CCL227-RH spheroids triggered CCID formation of underneath growing LEC and BEC monolayers significantly faster than naïve CCL227 spheroids (Fig. 1A and B). In detail, CCL227-RH spheroids time-dependently induced larger CCIDs than CCL227 spheroids in LECs $(15 \%$ after $0.5 \mathrm{~h}, \sim 35 \%$ after $1 \mathrm{~h}$ and $\sim 20 \%$ after $1.5 \mathrm{~h})$ and in BECs ( $\sim 15 \%$ after $0.5 \mathrm{~h}$ and $\sim 25 \%$ after $1 \mathrm{~h})$. 
A

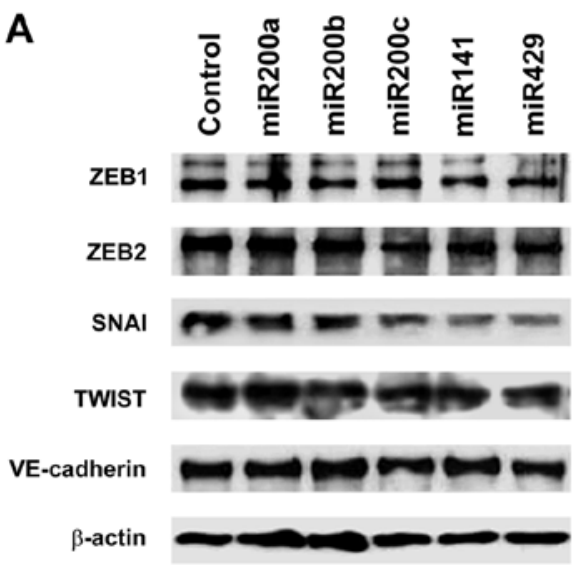

B CCL227-RH spheroids transfected with microRNA200

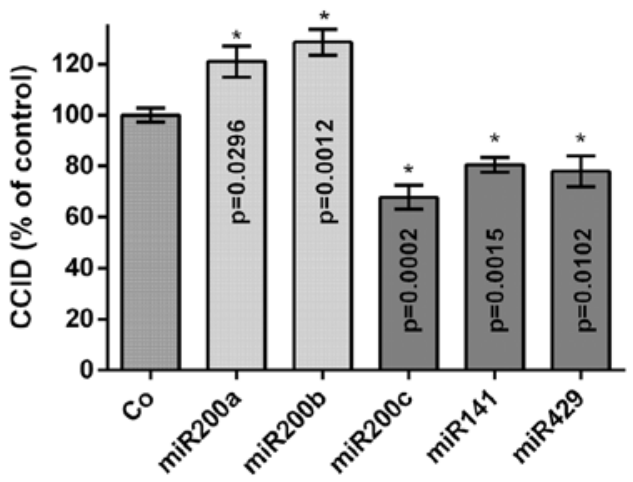

Figure 2. Effect of miR200 family members on the expression of EMT markers and CCID formation. (A) Expression of the EMT-related transcription factors ZEB1, ZEB2, SNAI, TWIST and of VE-cadherin in BECs, which were transfected with $5 \mathrm{nM}$ miR200 family precursors or non-targeting control, was analysed by western blotting. (B) 5-FU-resistant CCL227-RH spheroids were separately transfected with $5 \mathrm{nM}$ miR200a, miR200b, miR200c, miR141 and miR429 precursors or non-targeting control RNA $(\mathrm{Co})$ and placed on the BEC monolayer and CCID formation was measured; ( $=3$ ). Error bars indicate means \pm SEM and asterisks indicate significance ( $<<0.05$; Student's t-test). miR200, microRNA200; EMT, epithelial-to-mesenchymal transition; CCID, circular chemorepellent-induced defect; ZEB1, zinc finger E-box-binding homeobox 1; BECs, blood endothelial cells; 5-FU, 5-fluorouracil.

During the acquisition of 5-FU drug resistance CCL227-RH cells have lost the expression of miR200 family members, which are otherwise packed into exosomes and released from naïve CCL227 cells (4). Hence, exosomes derived from CCL227 cells are rich in miR200, whereas CCL227-RH exosomes are depleted (5). miR200 family members downregulate ZEBs, induce E-cadherin, reduce migration and limit EMT $(12,13)$. The downregulation of ZEB1 and upregulation of VE-cadherin in LECs was shown to correlate with reduced CCID formation triggered by MCF-7 spheroids (14). Therefore, we assumed that miR200-enriched exosomes, which are shed by CRC cells, act as potential modulators of BEC mobility and focussed on their effects on CCID formation in BEC monolayers. When naïve CCL227 spheroids were pre-incubated with exosomes derived from resistant CCL227-RH cells an increase of CCID formation $(20 \%)$ was observed after $4 \mathrm{~h}$ (Fig. 1C). We think that this effect was due to a dilution of miR200-containing exosomes of naïve CCL227 cells by miR200-depleted exosomes from 5-FU-resistant CCL227-RH cells. Reciprocally, the pre-incubation of CCL227-RH spheroids with exosomes collected from CCL227 cells, which are rich in miR200 $(4,5)$, reduced the CCID-forming potential of the CCL227-RH clone by 20\% (Fig. 1D) and this was most likely due to a concentration effect of miR200-enriched exosomes sticking to the spheroid surface.

The miR200 family inhibits ZEB transcription factor expression and CCID formation in BECs. Members of the miR200 family comprising miR200a, miR200b, miR200c, miR141 and miR429 regulate EMT (6). To examine which of these members impose the strongest effect on the ZEB family of transcription factors in BECs, $5 \mathrm{nM}$ miR precursors were separately transfected and the expression of ZEB1, ZEB2, SNAI, TWIST (SLUG was not expressed) and VE-cadherin was analysed by western blotting (Fig. 2A). The immunoblots indicated a repression of ZEB1, ZEB2, SNAI and TWIST upon transfection of all five miR200 family members. ZEB1 was repressed by miR200a and miR200b, TWIST was inhibited upon transfection with miR200c and miR141, whereas ZEB2 and SNAI were most effectively inhibited by miR200c, miR141 and miR429. VE-cadherin expression was almost unaffected in BECs and similar to that in LECs (4). Since CRC-derived miR200c, miR141 and miR429 were the strongest suppressors of ZEB2 and SNAI in BECs, we focussed on the stromal effects of these transcription factors and how this influenced CCID formation in the CRC/BEC model. For this, resistant CCL227-RH spheroids were transfected with the individual precursor microRNAs and placed on BEC monolayers. miR200c, miR141 and miR429 inhibited CCID formation significantly (30, 20 and 20\%, respectively), whereas miR200a and miR200b induced CCID formation (Fig. 2B). This suggested that inhibition of ZEB1 by miR200a and miR200b did not play a role in stabilising the BEC barriers, whereas miR200c, miR141 and miR429, which inhibited ZEB2 and SNAI in BECs, attenuated their disintegration.

Drug-dependent inhibition of CRC spheroid-triggered disintegration of BEC barriers. It was reported that loss of histone acetylation during CRC development correlates with advanced tumour stage and tumour invasion, because a reduction in global acetylation of histone $\mathrm{H} 4$ was observed in $80 \%$ of colon carcinomas and 39\% of adenomas (15). Hence, HDAC inhibitors are receiving increasing interest as chemopreventive and chemotherapeutic agents. With the goal to re-express miR200 family members and to reduce malignancy the CRC/BEC model was tested with the class I HDAC inhibitor mocetinostat and with the HDAC inhibitor sulforaphane, which is a natural secondary metabolite present mostly in cruciferous vegetables and in particularly high quantity in broccoli sprouts $(15,16)$. Thus, CCL227 and CCL227-RH spheroids were pre-treated with mocetinostat and sulforaphane for $20 \mathrm{~min}$ and then placed on BEC monolayers to analyse CCID formation. The migration of BECs, induced by CCL227 and CCL227-RH spheroids, was reduced by mocetinostat and sulforaphane in a dose-dependent manner. Both compounds were non-toxic 
A
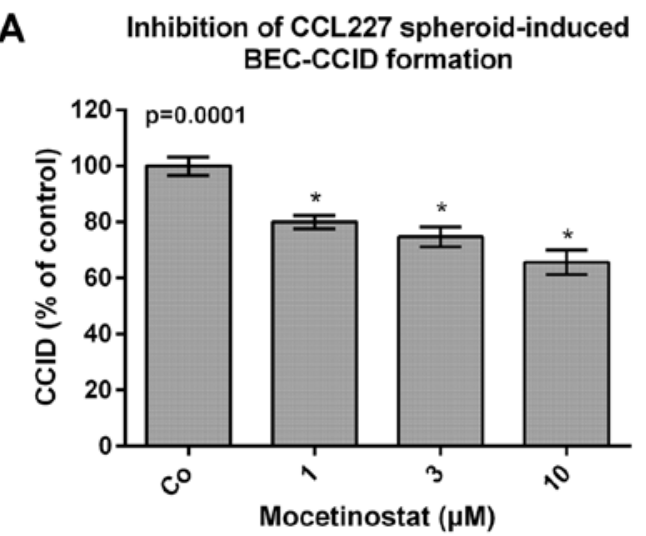

C Inhibition of CCL227 spheroid-induced BEC-CCID formation

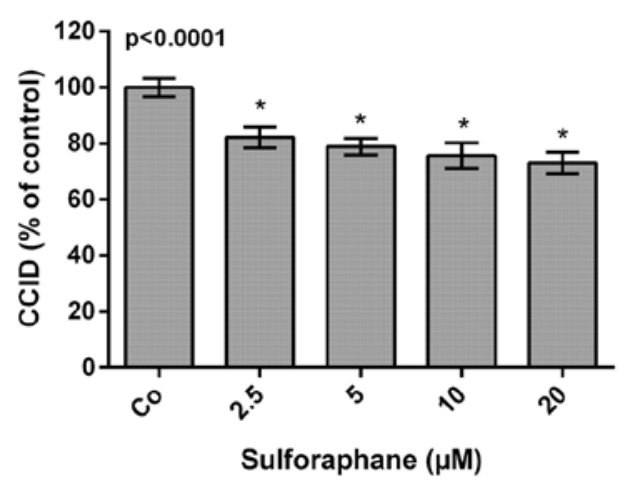

B

Inhibition of CCL227-RH spheroid-induced BEC-CCID formation

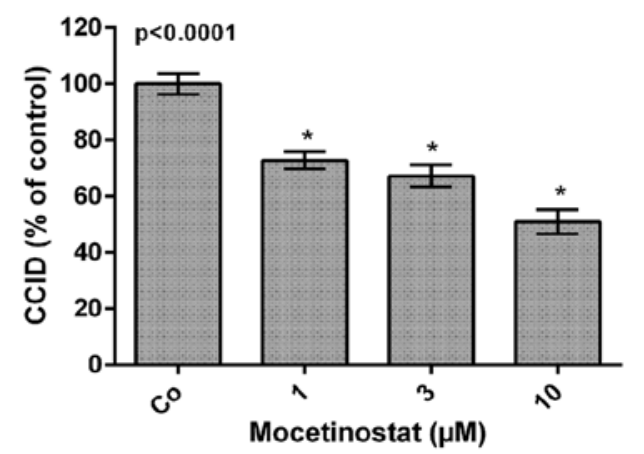

D Inhibition of CCL227-RH spheroid-induced BEC-CCID formation

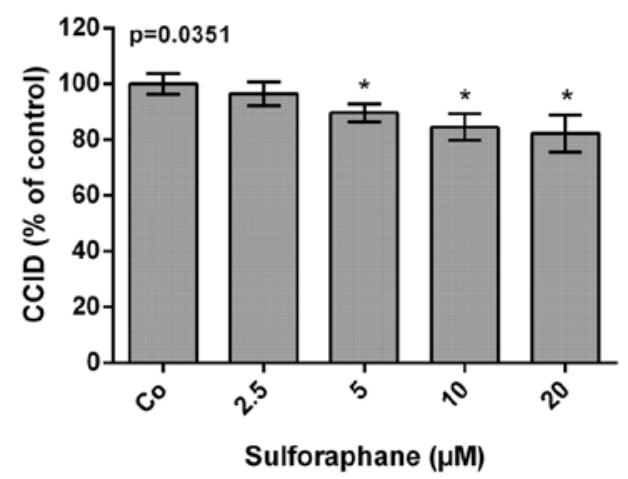

Figure 3. Inhibition of CCID formation by the HDAC inhibitors mocetinostat and sulforaphane. (A and C) Naïve CCL227 or (B and D) 5-FU-resistant CCL227-RH spheroids were placed on BEC monolayers and co-cultivated for $3 \mathrm{~h}$ either with medium alone and solvent (DMSO; Co) or with increasing concentrations of $(\mathrm{A}$ and $\mathrm{B})$ mocetinostat and $(\mathrm{C}$ and $\mathrm{D})$ sulforaphane when CCID areas underneath the spheroids were measured ( $\mathrm{n}=2 ; 10$ technical replicates). Error bars indicate means \pm SEM and asterisks indicate significance (one-way ANOVA; $<<0.05$ ). CCID, circular chemorepellent-induced defect; 5-FU, 5-fluorouracil; BEC, blood endothelial cell.

during the treatment time at the used concentrations (data not shown). Mocetinostat inhibited CCL227-RH-induced CCIDs more efficiently $(30,35$ and $40 \%)$ than CCIDs induced by CCL227 spheroids (20, 25 and 30\%) (Fig. 3A and B). In contrast, sulforaphane, which was less active than mocetinostat, inhibited CCL227-RH-induced CCID formation by only $20 \%$ and CCL227-induced CCIDs by 20-25\% (Fig. 3C and D).

\section{Discussion}

The present study investigated the response of BECs upon contact with naïve CRC spheroids or with spheroids consisting of high 5-FU-resistant CRC cells and confirmed earlier data showing that the mobility of LECs was increased in the neighbourhood of aggressive 5-FU-resistant CRC-RH cells as compared to less aggressive naïve CRC cells (4). Furthermore, the $\mathrm{CRC} / \mathrm{LEC}$ and $\mathrm{CRC} / \mathrm{BEC}$ in vitro models recapitulated the pathologic situation in which CRC spreads through lymphatics as well as through blood vessels. In contrast, it was demonstrated that LEC barriers were about five times more sensitive to breast cancer spheroid-induced CCID formation than BEC barriers resembling the metastatic route of breast cancer cells through lymphatics rather that blood vessels (2). Altogether, these findings underscore the validity of the three-dimensional (3D) CCID assay as a tool to study tumour breaching through the endothelial vasculature in vitro.
Recently, it was reported that CCL227 cells secrete exosomes containing miR200 family members whereas the exosomes of 5-FU-resistant CCL227-RH cells are virtually free of miR200, and that miR200c inhibits CCID formation in the CRC/LEC model (4). Here we demonstrate that miR200c, miR141 and miR429 (but not miR200a and miR200b) inhibit CCID formation also in the CRC/BEC model. In BECs miR200c, miR141 and miR429 inhibited ZEB2 and SNAI most effectively. SNAI plays a key role in invasion and metastases of CRC, which is driven by the TGF- $\beta 1 /$ SMAD signalling pathway (17). Furthermore, SNAI specifically represses vitamin D receptor, which itself mediates antitumour action and is lost in progressive CRC (18). Upon SNAI-induced EMT the synthesis of ZEB2 is upregulated (19) and miR132 (which does not belong to the miR200 family) inhibits CRC invasion and EMT by targeting ZEB2 (20). Hence, SNAI and ZEB2 significantly contribute to EMT, endothelial breaching and metastasis of CRC. Cells in the invasive front of colorectal tumours that have lost expression of the miR200 family members are more likely to intravasate into the blood or lymphatic system (6). Hence, malignant progression was attenuated through horizontal transfer of miR200c, miR141 and miR429 to an alien cell type. The downregulation of ZEBs upon transfection of miR200 family members into BECs was almost similar compared to LECs (4). As an exception, miR200a and miR200b did not influence ZEB2 expression 
and miR200c inhibited SNAI expression only weakly in LECs, whereas ZEB2 and SNAI were inhibited by these microRNAs in BECs. We cannot explain the inducing effect of miR200a and miR200b on CCID formation but it seems that gene regulation by these microRNAs is more complex than expected. Unlike the negative regulation of E-cadherin by ZEBs in epithelial cells, VE-cadherin was not suppressed by ZEBs in endothelial cells, which was supported by the fact that knockdown of ZEB1 did not influence the expression of VE-cadherin in LECs (14). Therefore, other mechanisms must have been involved in the regulation of VE-cadherin in BECs.

The class I HDAC inhibitor mocetinostat was shown to lead to a re-expression of miR200 family members which re-establishes sensitivity to gemcitabine-induced apoptosis of otherwise treatment-resistant pancreatic cancer cells (21). Acetylation/deacetylation events go hand in hand also with DNA methylations/demethylations, which was recently observed for the miR141 and miR200c loci in vitro and in vivo (22). Currently mocetinostat is studied in 17 clinical trials (https://clinicaltrials.gov/ct2/results?term=mocetinostat $\&$ Search=Search).

Sulforaphane is also reported to cause a global increase in histone $\mathrm{H} 3$ and $\mathrm{H} 4$ acetylation in HT-29 CRC cells similar to mocetinostat in pancreatic cancer cells (23). This led to the assumption that mocetinostat and sulforaphane might upregulate miR200 in BECs and influence CCID formation. The short treatment time with mocetinostat $(\sim 3.5 \mathrm{~h})$ seemed to exclude an epigenetic rearrangement of the chromatin as being causal for miR200 re-expression and for reduced aggressiveness. Nevertheless, it was shown that a single oral dose of sulforaphane was sufficient to inhibit HDAC activity and rearrange chromatin in mouse colon causing an increase in acetylated histones after $6 \mathrm{~h}$ (16) and the activation of the promoter regions of $p 21$ and Bax genes implicates epigenetic alterations within such a short treatment period (15). Hence, also short treatment time allows the re-expression of silenced genes. However, neither mocetinostat nor sulforaphane caused a significant change in the expression of miR200a, miR200b, miR200c, miR141 and miR429. Therefore, the inhibition of CCIDs upon treatment with mocetinostat and sulforaphane was independent of their anticipated effect on miR200 expression. Sulforaphane was shown in Caco-2 cells to exert also another pharmacologic activity, i.e., the induction of UGT1A1 and GSTA1 transcription (detoxification enzymes) (24). As the disintegration of BEC barriers in vitro was attenuated by sulforaphane, which can be consumed by eating broccoli sprouts in quantities that change the expression in human PBMCs in vivo (16) and suppresses tumorigenesis in rodents (15), and by mocetinostat currently tested in clinical trials, both compounds may provide a tool to support endothelial integrity. Whether miR200 expression has diagnostic/therapeutic impact regarding the development of drug resistance of $\mathrm{CRC}$ remains to be established.

\section{Acknowledgements}

We wish to thank Toni Jäger for preparing the figures. C.H.N. was supported by technology grant (TSA Doktorat) financed by the Austrian Federal Ministry of Science and Research (BMFW) in frame of Asea Uninet. The study was supported by a grant of the Herzfelder'sche Family Foundation to G.K.

\section{References}

1. de Krijger I, Mekenkamp LJM, Punt CJA and Nagtegaal ID: MicroRNAs in colorectal cancer metastasis. J Pathol 224: 438-447, 2011.

2. Kerjaschki D, Bago-Horvath Z, Rudas M, Sexl V, Schneckenleithner C, Wolbank S, Bartel G, Krieger S, Kalt R, Hantusch B, et al: Lipoxygenase mediates invasion of intrametastatic lymphatic vessels and propagates lymph node metastasis of human mammary carcinoma xenografts in mouse. J Clin Invest 121: 2000-2012, 2011.

3. Tentes IK, Schmidt WM, Krupitza G, Steger GG, Mikulits W, Kortsaris A and Mader RM: Long-term persistence of acquired resistance to 5-fluorouracil in the colon cancer cell line SW620. Exp Cell Res 316: 3172-3181, 2010.

4. Senfter D, Holzner S, Kalipciyan M, Staribacher A, Walzl A, Huttary N, Krieger S, Brenner S, Jäger W, Krupitza G, et al: Loss of miR-200 family in 5-fluorouracil resistant colon cancer drives lymphendothelial invasiveness in vitro. Hum Mol Genet 24: 3689-3698, 2015.

5. Mader RM, Wieser M, Berger W, Kalipciyan M, Hackl M, Steger GG and Grillari J: Relevance of microRNA modulation in chemoresistant colon cancer in vitro. Int J Clin Pharmacol Ther 49: 67-68, 2011.

6. Paterson EL, Kazenwadel J, Bert AG, Khew-Goodall Y, Ruszkiewicz A and Goodall GJ: Down-regulation of the miRNA-200 family at the invasive front of colorectal cancers with degraded basement membrane indicates EMT is involved in cancer progression. Neoplasia 15: 180-191, 2013.

7. Findlay VJ, Wang C, Watson DK and Camp ER: Epithelial-to-mesenchymal transition and the cancer stem cell phenotype: Insights from cancer biology with therapeutic implications for colorectal cancer. Cancer Gene Ther 21: 181-187, 2014.

8. Sánchez-Martínez R, Cruz-Gil S, Gómez de Cedrón M, Álvarez-Fernández M, Vargas T, Molina S, García B, Herranz J, Moreno-Rubio J, Reglero G, et al: A link between lipid metabolism and epithelial-mesenchymal transition provides a target for colon cancer therapy. Oncotarget 6: 38719-38736, 2015.

9. Schoppmann SF, Soleiman A, Kalt R, Okubo Y, Benisch C, Nagavarapu U,Herron GS and Geleff S: Telomerase-immortalized lymphatic and blood vessel endothelial cells are functionally stable and retain their lineage specificity. Microcirculation 11: 261-269, 2004.

10. Nguyen CH, Senfter D, Basilio J, Holzner S, Stadler S, Krieger S, Huttary N, Milovanovic D, Viola K, Simonitsch-Klupp I, et al: $\mathrm{NF}-\kappa \mathrm{B}$ contributes to MMP1 expression in breast cancer spheroids causing paracrine PAR 1 activation and disintegrations in the lymph endothelial barrier in vitro. Oncotarget 6: 39262-39275, 2015.

11. Madlener S, Saiko P, Vonach C, Viola K, Huttary N, Stark N, Popescu R, Gridling M, Vo NT, Herbacek I, et al: Multifactorial anticancer effects of digalloyl-resveratrol encompass apoptosis, cell-cycle arrest, and inhibition of lymphendothelial gap formation in vitro. Br J Cancer 102: 1361-1370, 2010.

12. Loboda A, Nebozhyn MV, Watters JW, Buser CA, Shaw PM, Huang PS, Van't Veer L, Tollenaar RA, Jackson DB, Agrawal D, et al: EMT is the dominant program in human colon cancer. BMC Med Genomics 4: 9, 2011.

13. Yuan D, Xia H, Zhang Y, Chen L, Leng W, Chen T, Chen Q, Tang Q, Mo X, Liu M, et al: $\mathrm{p}-\mathrm{Akt} / \mathrm{miR} 200$ signaling regulates epithelial-mesenchymal transition, migration and invasion in circulating gastric tumor cells. Int J Oncol 45: 2430-2438, 2014.

14. Vonach C, Viola K, Giessrigl B, Huttary N, Raab I, Kalt R, Krieger S, Vo TP, Madlener S, Bauer S, et al: NF- $\kappa B$ mediates the 12(S)-HETE-induced endothelial to mesenchymal transition of lymphendothelial cells during the intravasation of breast carcinoma cells. Br J Cancer 105: 263-271, 2011.

15. Myzak MC,Dashwood WM, Orner GA, Ho E and Dashwood RH: Sulforaphane inhibits histone deacetylase in vivo and suppresses tumorigenesis in Apc-minus mice. FASEB J 20: 506-508, 2006.

16. Myzak MC, Tong P, Dashwood WM, Dashwood RH and Ho E: Sulforaphane retards the growth of human PC-3 xenografts and inhibits HDAC activity in human subjects. Exp Biol Med (Maywood) 232: 227-234, 2007. 
17. Ji Q, Liu X, Han Z, Zhou L, Sui H, Yan L, Jiang H, Ren J, Cai J and Li Q: Resveratrol suppresses epithelial-to-mesenchymal transition in colorectal cancer through TGF- $\beta 1 /$ Smads signaling pathway mediated Snail/E-cadherin expression. BMC Cancer 15: $97,2015$.

18. Larriba MJ, Martín-Villar E, García JM, Pereira F, Peña C, de Herreros AG, Bonilla F and Muñoz A: Snail2 cooperates with Snaill in the repression of vitamin D receptor in colon cancer. Carcinogenesis 30: 1459-1468, 2009.

19. Beltran M, Puig I, Peña C, García JM, Alvarez AB, Peña R, Bonilla $\mathrm{F}$ and de Herreros AG: A natural antisense transcript regulates Zeb2/Sip1 gene expression during Snail1-induced epithelial-mesenchymal transition. Genes Dev 22: 756-769, 2008.

20. Zheng YB, Luo HP, Shi Q, Hao ZN, Ding Y, Wang QS, Li SB, Xiao GC and Tong SL: miR-132 inhibits colorectal cancer invasion and metastasis via directly targeting ZEB2. World J Gastroenterol 20: 6515-6522, 2014.
21. Meidhof S, Brabletz S, Lehmann W, Preca BT, Mock K, Ruh M, Schüler J, Berthold M, Weber A, Burk U, et al: ZEB1-associated drug resistance in cancer cells is reversed by the class I HDAC inhibitor mocetinostat. EMBO Mol Med 7: 831-847, 2015.

22. Díaz-Martín J, Díaz-López A, Moreno-Bueno G, Castilla MÁ, Rosa-Rosa JM, Cano A and Palacios J: A core microRNA signature associated with inducers of the epithelial-to-mesenchymal transition. J Pathol 232: 319-329, 2014

23. Dashwood RH and Ho E: Dietary histone deacetylase inhibitors: From cells to mice to man. Semin Cancer Biol 17: 363-369, 2007.

24. Svehlíková V, Wang S, Jakubíková J, Williamson G, Mithen R and Bao Y: Interactions between sulforaphane and apigenin in the induction of UGT1A1 and GSTA1 in CaCo-2 cells. Carcinogenesis 25: 1629-1637, 2004. 